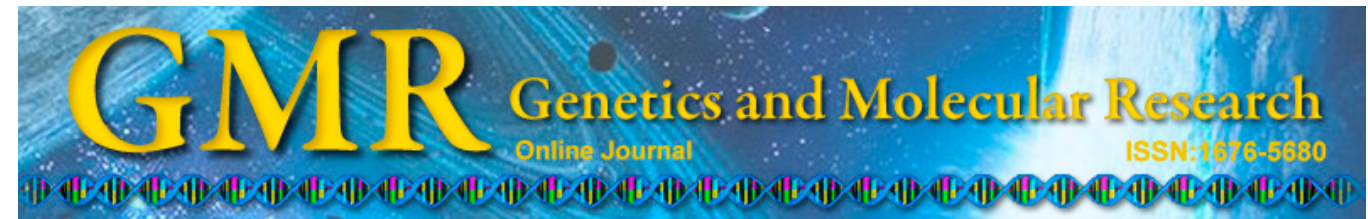

\title{
Association between polymorphisms in the thymidylate synthase gene and risk of breast cancer in a Mexican population
}

A. Quintero-Ramos ${ }^{1}$, S.A. Gutiérrez-Rubio ${ }^{1}$, A. Del Toro-Arreola ${ }^{1}$, R.A. Franco-Topete ${ }^{1,2}$, A. Oceguera-Villanueva ${ }^{3}$, L.M. Jiménez-Pérez ${ }^{4}$, J.M. Castro-Cervantes ${ }^{5}$, A. Barragán-Ruiz ${ }^{6}$, J.G. Vázquez-Camacho ${ }^{7}$ and A. Daneri-Navarro ${ }^{1}$

${ }^{1}$ Laboratorio de Inmunología, Centro Universitario de Ciencias de la Salud, Universidad de Guadalajara, Guadalajara, México

${ }^{2}$ Unidad de Anatomía Patológica, OPD Nuevo Hospital Civil de Guadalajara, Guadalajara, México

${ }^{3}$ Instituto Jalisciense de Cancerología, Secretaria de Salud Jalisco,

Guadalajara, México

${ }^{4}$ Departamento de Salud Pública, Centro Universitario de Ciencias de la Salud, Universidad de Guadalajara, Guadalajara, México

${ }^{5}$ Hospital de Especialidades, Centro Médico Nacional de Occidente, Instituto Mexicano del Seguro Social, Guadalajara, México

${ }^{6}$ Hospital de Gineco-Obstetricia, Centro Médico Nacional de Occidente, Instituto Mexicano del Seguro Social, Guadalajara, México

${ }^{7}$ Departamento de Anatomía Patológica, Centro Médico Nacional de Occidente, Instituto Mexicano del Seguro Social, Guadalajara, México

Corresponding author: A. Daneri-Navarro

E-mail: daneri@cucs.udg.mx

Genet. Mol. Res. 13 (4): 8749-8756 (2014)

Received October 7, 2013

Accepted May 14, 2014

Published October 27, 2014

DOI http://dx.doi.org/10.4238/2014.October.27.16

ABSTRACT. Breast cancer (BC) is the leading cause of cancer-related deaths among women in Mexico. Two single-nucleotide polymorphisms (SNPs) in the thymidylate synthase (TS) gene, the 28-base pair (bp) 
tandem repeat in the TS $5^{\prime}$-untranslated enhanced region (TSER) and the 6-bp insertion/deletion in the TS 3'-untranslated region (TS 3'-UTR), increase the rate of misincorporation of uridylate into DNA and may lead to chromosomal damage. We examined the association between these polymorphisms and $\mathrm{BC}$ risk in Mexican women according to menopause status. Mexican patients with initial BC diagnosis $(\mathrm{N}=230)$ and 145 individuals from a reference general population group (RGP) were included. For statistical analysis, the BC group was divided into pre- and post-menopause groups (PRE and POST groups, respectively). We analyzed both TS polymorphisms (TSER and TS 3'-UTR) using polymerase chain reaction. Finetti analysis was used to evaluate interand intra-group differences. The results showed a high frequency for the $3 \mathrm{R}$ and ins6 alleles in the BC, RGP, PRE, and POST groups. No significant differences were observed for the TS and TSER genotype and allele frequency distributions between groups. We found that the TSER and TS 3'-UTR SNPs are not associated with BC risk in Mexican patients.

Key words: Breast cancer; Thymidylate synthase; TS polymorphisms; TSER

\section{INTRODUCTION}

Breast cancer $(\mathrm{BC})$ is the leading cause of cancer mortality in Mexican women (Chávarri-Guerra et al., 2012). BC is a complex disease influenced by environmental and genetic factors, but most of these factors are unknown. Contributing factors include age, ethnicity, reproductive factors, hormones, familial history, lifestyle, and breast density (Henríquez-Hernández et al., 2009). Genetic alterations induced by endogenous or exogenous metabolites may also contribute to its etiology (Hashemi et al., 2012). Epidemiological studies have suggested that a folate-deficient diet may increase the risk of BC, but the results are inconsistent (Lajin et al., 2012). Folate is a water-soluble vitamin naturally found in green leafy vegetables, cereals, legumes, and fruits (Ronco et al., 2010). It is necessary for de novo synthesis of thymine, which is important in DNA synthesis, repair, integrity, and stability. Folate depletion alone can diminish the methyl pool. Furthermore, folate is the primary methyl group donor, and thus is very important in DNA methylation (Kim, 2000). Several key enzymes are involved in folate metabolism. Thymidylate synthase (TS) catalyzes the conversion of deoxiuridine monophosphate to deoxithymidine monophosphate and the conversion of 5,10-methylenetertahydrofolate to dihydrofolate (Ulrich et al., 2000). TS utilizes 5,10-methylenetetrahydrofolate as a methyl group donor to convert uracil to thymidine for DNA synthesis. Alterations in the one-carbon metabolism pathway can influence DNA methylation, synthesis, and repair, all of which play a critical role in carcinogenesis and thus may be involved in cancer development. Polymorphisms in critical enzymes involved in onecarbon metabolism, such as TS, play important and interrelated roles in folate metabolism, and thus may influence BC risk (Davis and Uthus, 2004; Suzuki et al., 2008). The TS gene is located in chromosome 18p11.32, and a tandem repeat polymorphism has been identified in 
the 5'-untranslated region (UTR) enhancer region of the TS promoter (TSER, rs34743033). TSER is located immediately upstream of the ATG codon initiation start site, which contains triple (TSER 3R) or double (TSER 2R) repeats of 28-bp sequences (Hitre et al., 2005). In vitro and in vivo studies have shown that TS expression is TSER-genotype dependent, and that the 3R allele is associated with a higher TS expression level (Kawakami et al., 1999). Another potentially functional polymorphism involving a 6-bp insertion/deletion (TAAAGT) at position 1494 in the TS 3'-UTR (rs16430) has been described. In addition, a deletion allele has been reported to result in low TS mRNA stability and low TS expression compared with the wild-type allele (Mandola et al., 2004). It has been reported that deficiencies in the thymidylate increase the rate of misincorporation of uridylate into DNA. These alterations produce DNA strand breaks and other chromosomal damage (Blount et al., 1997), and thus increase the predisposition to cancer. In this study, we examined the association between TS polymorphisms and $\mathrm{BC}$ development in Mexican women.

\section{MATERIAL AND METHODS}

\section{Subjects}

Patients were recruited as part of the Ella Binational Breast Cancer Study (Ella Study) from 3 public hospitals in Guadalajara, Jalisco, Mexico (Hospital de Especialidades-CMNOIMSS, Hospital de Gineco-Obstetricia-IMSS, and Instituto Jalisciense de Cancerologia). The Ella Study is a case-control study that includes women of Mexican descent in the United States and Mexico, and has been described previously (Martínez et al., 2010). In this study, we only included patients from the State of Jalisco, Mexico. A total of 230 Mexican women aged $\geq 18$ years with an initial diagnosis of $\mathrm{BC}$ that had been histologically confirmed were enrolled in the study. For analysis, the BC group was divided into two subgroups based on menopausal status, including premenopausal (PRE) and postmenopausal (POST) women. In addition, we analyzed a group of 145 individuals as a reference to the general population (RGP), or control group, which consisted of unrelated adults aged $\geq 18$ years, as representatives of those living in Guadalajara, Jalisco, Mexico.

\section{Ethical considerations}

The Institutional Review Board from each institution approved the study, and all study participants provided written informed consent.

\section{Genotyping}

DNA from each subject was extracted from leukocyte pellets using the Miller and ethanol precipitation methods (Miller et al., 1988). Genotyping was performed by polymerase chain reaction (PCR) using sequence-specific primers and 10 ng genomic DNA in a total volume of $10 \mu \mathrm{L}$, containing $1 \mathrm{X}$ PCR buffer, $1.5 \mathrm{mM} \mathrm{MgCl}, 100 \mathrm{M}$ of each dNTP, 1 pmol of each primer, and 0.05 U Taq polymerase (Invitrogen; Carlsbad, CA, USA). The pair of primers used for the TSER polymorphism was 5'-GTGGCTCCTGCGTTTCCCCC-3' (forward) and 5'-CTCCGAGCCGGCCACAGG-3' (reverse). PCR thermal conditions for TSER polymor- 
phisms were: initial denaturing at $94^{\circ} \mathrm{C}$ for $5 \mathrm{~min}, 33$ cycles of $94^{\circ} \mathrm{C}, 63^{\circ} \mathrm{C}$, and $72^{\circ} \mathrm{C}$ each for $30 \mathrm{~s}$, followed by final extension at $72^{\circ} \mathrm{C}$ for $10 \mathrm{~min}$ (Morganti et al., 2005). The TS 3'-UTR locus primers were 5'-TGATGTAGAGTGTGGTTATGAAC-3' (forward) and 5'-ATTATAGCA ACATATAAAACAAC-3' (reverse). PCR thermal conditions for the TS 3'-UTR polymorphisms were initial denatured at $94^{\circ} \mathrm{C}$ for $5 \mathrm{~min}, 30$ cycles of $94^{\circ}, 57^{\circ}$, and $72^{\circ} \mathrm{C}$ each for $35 \mathrm{~s}$, followed by a final extension at $72^{\circ} \mathrm{C}$ for $10 \mathrm{~min}$ (Zhang et al., 2005). PCR products were immediately electrophoresed on $6 \%$ polyacrylamide gels and visualized by silver staining. Allele identification was conducted using a 10-bp molecular weight standard (Invitrogen). The same assay was repeated for more than $10 \%$ of the samples for quality control; the concordance rate was $100 \%$ for the 2 polymorphisms in this study. Sample size variations between polymorphisms occurred due to PCR failure in some individuals.

\section{Statistical analysis}

The sample size was sufficient to identify differences in common allele ( $>5 \%)$ frequencies with a level of significance of 0.05 and a power of 0.80 (Chakraborty, 1992). For intra-group analysis, patients were stratified by menopausal status into 2 subgroups, PRE and POST. Genetic comparisons were made between all categories per variable. Allele frequencies were calculated by counting the genotypes. Observed genotype proportions were compared with Hardy-Weinberg Equilibrium (HWE) expectations based on the method of Finetti (Cannings and Edwards, 1968) and using $\chi^{2}$ tests. Inter- and intra-group genotype, phenotype, and allele distributions were compared using an interactive computer program also based on $\chi^{2}$ and exact tests (RXC for DOS program). The probability estimation of haplotype frequencies was calculated by using an algorithm method with the Arlequin software program V.3.11.

\section{RESULTS}

A total of 230 Mexican BC patients were recruited and their DNA was analyzed for the TSER and TS 3'-UTR polymorphisms. BC patients were classified according to hormonal status as either PRE (53 cases) or POST (143 cases). Because menopausal status was unknown for some samples, these were excluded from the analysis.

Genotype frequencies in the RGP group were in HWE, with $\mathrm{P}=0.95$ and $\mathrm{P}=0.59$, for the TSER and TS 3'-UTR polymorphisms, respectively. Allele and genotype frequencies for the TSER and TS 3'-UTR polymorphisms for the populations studied (RGP, BC, PRE, and POST groups) are shown in Table 1. We observed that the ins6 and 3R alleles and genotypes ins6/del6 and 2R/3R were observed most frequently in all groups for the TS 3'-UTR and TSER polymorphisms, respectively.

Because the alleles that confer a risk of breast cancer are $2 \mathrm{R}$ and del6 for the TSER and TS 3'-UTR polymorphisms, respectively, we analyzed their allelic frequencies. TSER allelic frequencies were 0.40 and 0.38 for the RGP and BC groups, respectively. TS 3'-UTR allelic frequencies were 0.30 and 0.31 for the RGP and $\mathrm{BC}$ groups, respectively.

Genotypes related to breast cancer risk include $2 \mathrm{R} / 2 \mathrm{R}$ for the TSER and del6/del6 for the TS 3'-UTR polymorphisms. Genotype frequencies for the TSER polymorphisms were 0.158 and 0.130 for RGP and BC, respectively. For TS 3'-UTR polymorphisms, genotype frequencies were 0.970 and 0.108 for RGP and BC, respectively. We observed that the allele and 
genotype frequencies were very similar among the different study groups and that the $\mathrm{BC}$ risk associations did not differ $(\mathrm{P}>0.05)$ with respect to menopausal status (PRE/POST, Table 2$)$. No significant associations were observed between the 2 polymorphisms and $\mathrm{BC}$ risk.

Table 1. Genotype and allelic frequencies of TSER and TS polymorphisms in the groups studied.

\begin{tabular}{lccrr}
\hline \multicolumn{3}{c}{ Group } \\
\hline TSER & RGP $(\mathrm{N}=145)[\mathrm{N},(\%)]$ & $\mathrm{BC}(\mathrm{N}=230)[\mathrm{N},(\%)]$ & PRE $(\mathrm{N}=53)[\mathrm{N},(\%)]$ & POST $(\mathrm{N}=143)[\mathrm{N},(\%)]$ \\
2R/2R & $23(15.86)$ & $30(13.04)$ & $5(9.43)$ & $19(13.29)$ \\
2R/3R & $69(47.59)$ & $116(50.43)$ & $27(50.94)$ & $69(48.25)$ \\
3R/3R & $53(36.55)$ & $84(36.52)$ & $21(36.62)$ & $55(38.46)$ \\
2R & $115(40)$ & $176(38)$ & $37(34.91)$ & $107(37.41)$ \\
3R & $175(60)$ & $284(62)$ & $69(65.09)$ & $179(62.59)$ \\
TS 3'UTR & RGP $(\mathrm{N}=165)[\mathrm{N},(\%)]$ & $\mathrm{BC}(\mathrm{N}=250)[\mathrm{N},(\%)]$ & $\mathrm{PRE}(\mathrm{N}=55)[\mathrm{N},(\%)]$ & $\mathrm{POST}(\mathrm{N}=155)[\mathrm{N},(\%)]$ \\
del6/del6 & $16(9.70)$ & $27(10.80)$ & $7(12.73)$ & $17(10.97)$ \\
del6/ins6 & $66(40)$ & $102(40.80)$ & $23(41.82)$ & $67(42.23)$ \\
ins6/ins6 & $83(50.30)$ & $121(48.40)$ & $25(45.45)$ & $71(45.81)$ \\
del6 & $98(30)$ & $156(31)$ & $37(33.64)$ & $101(32.58)$ \\
ins6 & $232(70)$ & $344(69)$ & $73(66.36)$ & $209(67.42)$ \\
\hline
\end{tabular}

$\mathrm{RGP}=$ reference general population group; $\mathrm{BC}=$ breast cancer group; $\mathrm{PRE}=$ premenopausal group; $\mathrm{POST}=$ postmenopausal group.

Table 2. Exact test $\mathrm{P}$ values and OR comparisons of groups studied.

\begin{tabular}{|c|c|c|c|c|}
\hline & Genotype $\mathrm{P}\left(\chi^{2}\right)$ & Allele $\mathrm{P}\left(\chi^{2}\right)$ & Phenotype $\mathrm{P}\left(\chi^{2}\right)$ & $\mathrm{OR}(\mathrm{CI})$ \\
\hline \multicolumn{5}{|l|}{ TSER } \\
\hline $\mathrm{RGP} v s \mathrm{BC}$ & $0.72(0.65)$ & $0.70(0.15)$ & $0.99(0.00)$ & $1.00(0.65-1.54)$ \\
\hline RGP vs PRE & $0.52(1.32)$ & $0.39(0.74)$ & $0.25(1.32)$ & $1.81(0.65-5.04)$ \\
\hline RGP vs POST & $0.82(0.41)$ & $0.58(0.31)$ & $0.74(0.11)$ & $0.92(0.57-1.49)$ \\
\hline $\mathrm{BC} v s$ PRE & $0.75(0.57)$ & $0.52(0.41)$ & $0.67(0.18)$ & $0.88(0.48-1.62)$ \\
\hline $\mathrm{BC} v s$ POST & $0.92(0.18)$ & $0.81(0.05)$ & $0.71(0.14)$ & $0.92(0.59-1.42)$ \\
\hline PRE $v s$ POST & $0.76(0.21)$ & $0.64(0.21)$ & $0.88(0.02)$ & $1.50(0.55-2.00)$ \\
\hline TS 3'UTR & Genotypic & Allelic & Phenotypic & OR (CI) \\
\hline $\mathrm{RGP} v s \mathrm{BC}$ & $0.90(0.21)$ & $0.65(0.21)$ & $0.70(0.14)$ & $1.08(0.73-1.60)$ \\
\hline RGP vs PRE & $0.74(0.59)$ & $0.48(0.60)$ & $0.53(0.39)$ & $1.22(0.66-2.24)$ \\
\hline RGP vs POST & $0.72(0.66)$ & $0.43(0.62)$ & $0.42(0.65)$ & $1.19(0.77-1.86)$ \\
\hline $\mathrm{BC} v s \mathrm{PRE}$ & $0.89(0.24)$ & $0.62(0.25)$ & $0.69(0.16)$ & $1.12(0.63-2.02)$ \\
\hline BC vs POST & $0.87(80.23)$ & $0.68(0.17)$ & $0.61(0.26)$ & $1.11(0.74-1.66)$ \\
\hline PRE $v s$ POST & $0.94(0.13)$ & $0.84(0.04)$ & $0.96(0.00)$ & $0.99(0.53-1.83)$ \\
\hline
\end{tabular}

$\mathrm{RGP}=$ reference general population group; $\mathrm{BC}=$ breast cancer group; $\mathrm{PRE}=$ premenopausal group; POST $=$ postmenopausal group. Phenotype is formed for del6/del6 + del6/ins6 to TS 3'-UTR polymorphisms and 2R/2R + 2R/3R for TSER polymorphisms. OR was calculated for phenotype.

Although we did not observe differences in our analysis of a $2 \mathrm{R}$ dominant phenotype formed by $2 R / 2 R+2 R / 3 R$ for TSER, we found an elevated odds ratio (OR) value between the RGP and the PRE groups $\left(\mathrm{P}=0.25, \chi^{2}=1.32, \mathrm{OR}=1.81\right.$, confidence interval $(\mathrm{CI})=0.65$ 5.04). Similarly, for the del6 dominant phenotype del6/del6 + del6/ins6 for the TS 3'-UTR polymorphisms, we observed an elevated OR value between RGP and PRE ( $\mathrm{P}=0.53, \chi^{2}=$ $0.39, \mathrm{OR}=1.22, \mathrm{CI}=0.66-2.24$, Table 2 ). Only in the comparison of RGP versus PRE, we found OR values to be higher, in contrast to the comparison of other groups.

The haplotype ins6/3R showed a relatively high frequency in the 4 groups studied, while the haplotype del6/2R was less frequent in the 4 groups. Comparison of the observed 
frequencies of the 4 study groups showed no significant difference (Table 3). Linkage disequilibrium for the different groups was low, less than 0.02 , indicating that the 2 polymorphisms analyzed are very distant from each other.

Table 3. Haplotype frequencies for TS 3'-UTR and TSER polymorphisms in the different subgroups.

\begin{tabular}{lcccc}
\hline & \multicolumn{4}{c}{ Group } \\
\cline { 2 - 5 } TS and TSER haplotypes & RGP $(4 \mathrm{~N}=580), \mathrm{N}(\%)$ & $\mathrm{BC}(4 \mathrm{~N}=1105), \mathrm{N}(\%)$ & PRE $(4 \mathrm{~N}=208), \mathrm{N}(\%)$ & POST $(4 \mathrm{~N}=540), \mathrm{N}(\%)$ \\
\hline del6-2R & $55(9.48)$ & $110(9.95)$ & $20(9.62)$ & $51(9.44)$ \\
del6-3R & $113(19.48)$ & $206(18.64)$ & $50(24.04)$ & $133(24.63)$ \\
Ins6-2R & $175(30.17)$ & $393(35.57)$ & $54(25.96)$ & $153(28.33)$ \\
Ins6-3R & $237(40.86)$ & $396(35.84)$ & $84(40.38)$ & $203(37.59)$ \\
$\mathrm{D}^{\prime}$ & 0.0114 & 0.0154 & 0.0126 & 0.0172 \\
$\mathrm{P}\left(\chi^{2}\right)$ & RGP $v$ BC & RGP $v$ s PRE & RGP $v$ POST & BC vs PRE \\
& $0.11(6.07)$ & $0.48(2.48)$ & $0.22(4.46)$ & $0.65(0.37)$ \\
& BC $v$ POST & PRE $v s$ POST & & \\
\hline
\end{tabular}

$\mathrm{RGP}=$ reference general population group; $\mathrm{BC}=$ breast cancer group; $\mathrm{PRE}=$ premenopausal group; $\mathrm{POST}=$ postmenopausal group. $\mathrm{D}^{\prime}=$ linkage disequilibrium.

\section{DISCUSSION}

$\mathrm{BC}$ is a complex multifactorial disease caused by both genetic and environmental factors. Enzymes that participate in the one-carbon metabolic route, which take part during the methylation, synthesis, and repair of DNA, are altered in cancer. We hypothesized that one-carbon metabolism plays an important role in $\mathrm{BC}$ pathogenesis. Genetic variations in enzymes involved in one-carbon metabolism are good candidates for examining the impact of their effects and interactions on $\mathrm{BC}$ risk. Epidemiological evidence suggests that the risk of $\mathrm{BC}$ may be reduced by increasing the consumption of folate and related B vitamins (Zhang et al., 2011; Yang et al., 2013). In the present study, we investigated whether the TSER and TS 3'-UTR polymorphisms in the TS gene, a molecule that plays a key role in DNA synthesis, are associated with the risk of $\mathrm{BC}$ development. This is the first study in Mexico to evaluate genetic variations involved in the one-carbon metabolism pathway in relation to breast cancer risk. Because of the high prevalence of these polymorphisms and the increasing incidence of $\mathrm{BC}$ in Mexico, the results of our study are important for determining the role of TS gene polymorphisms in $\mathrm{BC}$ risk.

Studies in other populations have found significant associations between the TS gene 5'-UTR and TSER variations and BC development (Wang et al., 2011; da Silva Nogueira et al., 2012). The 3R allele of the TSER 3'-UTR polymorphism is known to enhance TS enzymatic activity (Kawakami et al., 1999). Therefore, the 2R/3R genotype should show intermediate activity. The 3R/3R genotype for the TS gene has been associated with high levels of enzyme activity in tumors. Because TS competes with methylenetetrahydrofolate reductase during folic acid metabolism for the availability of 5,10-methylenetetrahydrofolate, the $3 \mathrm{R}$ variant may affect levels of 5,10-methylenetetrahydrofolate, leading to a lower cellular concentration of $S$-adenosylmethionine and a subsequent decrease in DNA methylation (Trinh et al., 2002). Thus, DNA hypomethylation may increase the susceptibility to genetic mutations and alter the expression of genes such as proto-oncogenes or tumor suppressor genes. This may result in epigenetic changes that initiate carcinogenesis, and subsequently cancer. However, in our 
study, differences in the allele frequency for the RGP and BC groups were not significant $(\mathrm{P}=$ 0.70 ), with a similar trend observed in both populations. The $3 \mathrm{R} / 3 \mathrm{R}$ genotypic frequencies for the RGP and $\mathrm{BC}$ groups were not significantly different $(\mathrm{P}=0.72)$. Allele, genotype, and phenotype frequencies in the PRE and POST groups were also not significantly different $(\mathrm{P}>0.5)$. Our results are very similar to those reported for Spanish women (Henríquez-Hernández et al., 2009, 2010), suggesting the importance of ethnic factors in determining BC susceptibility.

Recent studies have investigated the association between the TS 3'-UTR del6 polymorphism and risk of several types of cancer, including breast cancer; however, the results have been inconsistent (Trinh et al., 2002; Henríquez-Hernández et al., 2009, 2010; Wang et al., 2011; Zhou et al., 2012). In the present study, we found that the TS 3'-UTR del6 allele showed a small increase in both allelic and genotypic frequencies in the PRE group, but this increase was not significant $(\mathrm{P}>0.05)$. Our findings do not support the hypothesis that TS gene polymorphisms are useful genetic markers for assessing BC risk in the Mexican population. Discrepancies between the studies examining this variant may be related to several factors (da Silva Nogueira et al., 2012): heterogeneity of BC molecular subtypes, population ethnic backgrounds, uncharacterized environmental exposure factors, and/or potential biases due to small sample sizes.

Other studies suggest that the TSER polymorphism increases the susceptibility to BC in the Caucasian population, and the TS 3'-UTR polymorphism has been proposed as a genetic determinant for developing BC in the Asian population (Wang et al., 2011; Zhou et al., 2012). Our study is the first to correlate BC and polymorphisms in the 5'-UTR region of the TS gene in a Mexican population. Therefore, it is difficult to compare our results with other study populations with similar characteristics.

We observed that the two polymorphisms analyzed segregate independently; therefore, the probability of observing them together is very low. The prevalence of allelic polymorphisms 3R and del6 for the TSER and TS 3'UTR polymorphism, respectively, are increased compared with the $2 \mathrm{R}$ and ins 6 alleles in our population. Finally, the polymorphisms analyzed were not associated with the risk of $\mathrm{BC}$ in the Mexican population.

\section{ACKNOWLEDGMENTS}

The authors are indebted to Lizette Lorenz for proofreading the manuscript. Research supported by the Avon Foundation and Arizona Cancer Center Core Grant from the National Cancer Institute (\#CA-023074-2953).

\section{REFERENCES}

Blount BC, Mack MM, Wehr CM, MacGregor JT, et al. (1997). Folate deficiency causes uracil misincorporation into human DNA and chromosome breakage: implications for cancer and neuronal damage. Proc. Natl. Acad. Sci. U. S. A. 94: 3290-3295.

Cannings C and Edwards AW (1968). Natural selection and the de Finetti diagram. Ann. Hum. Genet. 31: 421-428.

Chakraborty R (1992). Multiple alleles and estimation of genetic parameters: computational equations showing involvement of all alleles. Genetics 130: 231-234.

Chávarri-Guerra Y, Villarreal-Garza C, Liedke PE, Knaul F, et al. (2012). Breast cancer in Mexico: a growing challenge to health and the health system. Lancet Oncol. 13: e335-e343.

da Silva Nogueira J Jr, de Lima Marson FA and Sílvia Bertuzzo C (2012). Thymidylate synthase gene (TYMS) polymorphisms in sporadic and hereditary breast cancer. BMC Res. Notes 5: 676. 
Davis CD and Uthus EO (2004). DNA methylation, cancer susceptibility, and nutrient interactions. Exp. Biol. Med. 229: 988-995.

Hashemi M, Eskandari-Nasab E, Fazaeli A, Taheri M, et al. (2012). Association between polymorphisms of glutathione S-transferase genes (GSTM1, GSTP1 and GSTT1) and breast cancer risk in a sample Iranian population. Biomark. Med. 6: 797-803.

Henríquez-Hernández LA, Murias-Rosales A, Hernández González A, Cabrera De León A, et al. (2009). Gene polymorphisms in TYMS, MTHFR, p53 and MDR1 as risk factors for breast cancer: a case-control study. Oncol. Rep. 22: 1425-1433.

Henríquez-Hernández LA, Perez LF, Hernández González A, Cabrera De León A, et al. (2010). TYMS, MTHFR, p53 and MDR1 gene polymorphisms in breast cancer patients treated with adjuvant therapy. Cancer Epidemiol. 34: 490-493.

Hitre E, Budai B, Adleff V, Czeglédi F, et al. (2005). Influence of thymidylate synthase gene polymorphisms on the survival of colorectal cancer patients receiving adjuvant 5-fluorouracil. Pharmacogenet. Genomics 15: 723-730.

Kawakami K, Omura K, Kanehira E and Watanabe Y (1999). Polymorphic tandem repeats in the thymidylate synthase gene is associated with its protein expression in human gastrointestinal cancers. Anticancer Res. 19: 3249-3252.

Kim YI (2000). Methylenetetrahydrofolate reductase polymorphisms, folate, and cancer risk: a paradigm of gene-nutrient interactions in carcinogenesis. Nutr. Rev. 58: 205-209.

Lajin B, Alhaj SA, Ghabreau L and Alachkar A (2012). Association of polymorphisms in one-carbon metabolizing genes with breast cancer risk in Syrian women. Tumour Biol. 33: 1133-1139.

Mandola MV, Stoehlmacher J, Zhang W, Groshen S, et al. (2004). A 6 bp polymorphism in the thymidylate synthase gene causes message instability and is associated with decreased intratumoral TS mRNA levels. Pharmacogenetics 14: 319-327.

Martínez ME, Gutiérrez-Millan LE, Bondy M, Daneri-Navarro A, et al. (2010). Comparative study of breast cancer in Mexican and Mexican-American women. Health 2: 1040-1048.

Miller SA, Dykes DD and Polesky HF (1988). A simple salting out procedure for extracting DNA from human nucleated cells. Nucleic Acids Res. 16: 1215.

Morganti M, Ciantelli M, Giglioni B, Putignano AL, et al. (2005). Relationships between promoter polymorphisms in the thymidylate synthase gene and mRNA levels in colorectal cancers. Eur. J. Cancer 41: 2176-2183.

Ronco AL, De Stefani E, Deneo-Pellegrini H, Boffetta P, et al. (2010). Dietary patterns and risk of ductal carcinoma of the breast: a factor analysis in Uruguay. Asian Pac. J. Cancer Prev. 11: 1187-1193.

Suzuki T, Matsuo K, Hirose K, Hiraki A, et al. (2008). One-carbon metabolism-related gene polymorphisms and risk of breast cancer. Carcinogenesis 29: 356-362.

Trinh BN, Ong CN, Coetzee GA, Yu MC, et al. (2002). Thymidylate synthase: a novel genetic determinant of plasma homocysteine and folate levels. Hum. Genet. 111: 299-302.

Ulrich CM, Bigler J, Velicer CM, Greene EA, et al. (2000). Searching expressed sequence tag databases: discovery and confirmation of a common polymorphism in the thymidylate synthase gene. Cancer Epidemiol. Biomarkers Prev. 9: 1381-1385.

Wang J, Wang B, Bi J and Di J (2011). The association between two polymorphisms in the TYMS gene and breast cancer risk: a meta-analysis. Breast Cancer Res. Treat. 128: 203-209.

Yang D, Baumgartner RN, Slattery ML, Wang C, et al. (2013). Dietary intake of folate, B-vitamins and methionine and breast cancer risk among Hispanic and non-Hispanic white women. PLoS One 8: e54495.

Zhang CX, Ho SC, Chen YM, Lin FY, et al. (2011). Dietary folate, vitamin B6, vitamin B12 and methionine intake and the risk of breast cancer by oestrogen and progesterone receptor status. Br. J. Nutr. 106: 936-943.

Zhang Z, Xu Y, Zhou J, Wang X, et al. (2005). Polymorphisms of thymidylate synthase in the 5'- and 3'-untranslated regions associated with risk of gastric cancer in South China: a case-control analysis. Carcinogenesis 26: 1764-1769.

Zhou JY, Shi R, Yu HL, Zeng Y, et al. (2012). The association between two polymorphisms in the TS gene and risk of cancer: a systematic review and pooled analysis. Int. J. Cancer 131: 2103-2116. 